\title{
miR-203 Functions as a Tumor Suppressor by Inhibiting Epithelial to Mesenchymal Transition in Ovarian Cancer
}

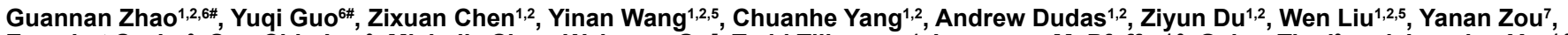
Erzsebet Szabo ${ }^{3}$, Sue-Chin Lee ${ }^{3}$, Michelle Sims, Weiwang Gu ${ }^{5}$, Todd Tillmanns ${ }^{4}$, Lawrence M. Pfeffer ${ }^{1,2}$, Gabor Tigyi ${ }^{3}$ and Junming Yue ${ }^{1,2}$

${ }^{1}$ Department of Pathology and Laboratory Medicine, University of Tennessee Health Science Center, USA

${ }^{2}$ Center for Cancer Research, University of Tennessee Health Science Center, Memphis, TN, USA

${ }^{3}$ Department of Physiology, University of Tennessee Health Science Center, USA

${ }^{4}$ West Cancer Center, Memphis, TN, USA

${ }^{5}$ Southern Medical University, Guangzhou, China

${ }^{6}$ The Third Affiliated Hospital, Zhengzhou University, China

${ }^{7}$ Harbin Medical University, P. R. China

\#Contributed equally

\section{Abstract}

Objective: Ovarian cancer is a gynecological malignancy that has a high mortality rate in women due to metastatic progression and recurrence. miRNAs are small, endogenous, noncoding RNAs that function as tumor suppressors or oncogenes in various human cancers by selectively suppressing the expression of target genes. The objective of this study is to investigate the role of miR-203 in ovarian cancer.

Methods: miR-203 was expressed in ovarian cancer SKOV3 and OVCAR3 cells using lentiviral vector and cell proliferation, migration, invasion were examined using MTT, transwell and Matrigel assays, respectively. Tumor growth was examined using Xenograft mouse model.

Results: miR-203 expression was downregulated, whereas expression of its target gene Snai2 was upregulated in human ovarian serous carcinoma tissue as compared to normal ovaries. In addition, high miR203 expression was associated with long-term survival rate of ovarian cancer patients. miR-203 overexpression inhibited cell proliferation, migration, and invasion of SKOV3 and OVCAR3 ovarian cancer cells. Furthermore, miR-203 overexpression inhibited the epithelial to mesenchymal transition (EMT) in ovarian cancer cells. Silencing Snai2 with lentiviral short hairpin (sh) RNA mimics miR-203-mediated inhibition of EMT and tumor cell invasion. Xenografts of miR-203-overexpressing ovarian cancer cells in immunodeficient mice exhibited a significantly reduced tumor growth.

Conclusion: miR-203 functions as a tumor suppressor by down regulating Snai2 in ovarian cancer.

Keywords: miR-203; Epithelial to mesenchymal transition; Ovarian cancer

\section{Introduction}

Ovarian cancer has a high mortality rate, with over 20,000 new cases diagnosed and 15,000 deaths every year in the US [1]. The high mortality rate in ovarian cancer reflects late stage diagnosis, disease recurrence, and chemoresistance [2,3]. However, the underlying molecular mechanisms for metastatic progression and chemoresistance of ovarian cancer remain unclear. The cellular phenotypic switch from epithelial to mesenchymal cell transition (EMT) is a fundamental process in tumor initiation, progression, metastasis, and chemoresistance [48]. The EMT process is accompanied by alterations of epithelial and mesenchymal marker genes, including Snail and 2, Zeb 1 and 2, Twist1 and 2, vimentin, and E-cadherin [9-11].

At initial diagnosis a late stage of metastatic progression is often observed in ovarian cancer, because of direct tumor extension and cell spreading directly through the peritoneal fluid with resultant invasion to adjacent organs [12]. Recent studies strongly support the role of EMT in ovarian cancer metastasis and chemoresistance [13-15]. A spheroid model has been proposed to address the role of EMT in ovarian cancer [16]. The rationale is that epithelial ovarian cancer cells undergo EMT and shed tumor cells into the peritoneum, which then survive as cellular aggregates or spheroids in the ascites. Subsequently, the spheroids attach themselves to the omentum, invade adjacent organs and undergo EMT/mesenchymal to epithelial cell transition
(MET) interconversion to enhance cell proliferation and progression [16]. Following EMT/MET interconversion during the metastatic progression, E-cadherin is upregulated from primary to secondary metastatic sites, suggesting that MET is required for the survival signaling pathways of tumor cells. EMT is one of the key drivers of chemoresistance and metastasis of ovarian cancer [14,17,18]. Signaling pathways, including WNT, Jak2/Stat3, PI3 kinase/AKT, and Notch, are found to regulate EMT [19-23].

miRNAs are a class of endogenous, non-coding, small RNAs that

*Corresponding authors: Junming Yue, University of Tennessee Health Science Center, 19 S. Manassas St., Rm. 266, Memphis, TN 38163; USA, Tel: 901-4482091; Fax: 901-448-3910; E-mail: jyue@uthsc.edu

Lawrence Pfeffer, University of Tennessee Health Science Center, 19 S. Manassas St, Rm.154, Memphis, TN 38163; USA, Tel: 901-448-7855; Fax: 901-448-3910; E-mail: Ipfeffer@uthsc.edu

Received December 18, 2014; Accepted February 03, 2015; Published February 06, 2015

Citation: Zhao G, Guo Y, Chen Z, Yang Y, Yang C, et al. (2015) miR-203 Functions as a Tumor Suppressor by Inhibiting Epithelial to Mesenchymal Transition in Ovarian Cancer. J Cancer Sci Ther 7: 034-043. doi:10.4172/1948-5956.1000322

Copyright: @ 2015 Zhao G, et al. This is an open-access article distributed under the terms of the Creative Commons Attribution License, which permits unrestricted use, distribution, and reproduction in any medium, provided the original author and source are credited. 
suppress protein translation at the posttranscriptional level and function as tumor suppressors and oncogenes in human cancers. Recent studies indicate that miRNAs, such as miR-194, miR-181a, miR-429, miR101, miR-506, and miR-200c, regulate EMT by directly targeting the epithelial or mesenchymal cell marker genes [11,24-27]. miRNA-based EMT profile is well correlated with ovarian cancer development and survival rate [12]. miR-203 has been reported to function as a tumor suppressor in lung [28], prostate [29], and breast cancer [30]. miR-203 expression was found to be upregulated and associated with advanced tumor progression and poor prognosis in ovarian cancer [31]. In the present study, we demonstrated that miR-203 expression was reduced in ovarian cancer patient samples. Moreover, miR-203 functions as a tumor suppressor in ovarian cancer, and inhibits ovarian cancer cell proliferation, migration, and invasion as well as tumor growth in a xenograft mouse model by directly targeting the mesenchymal marker Snai2.

\section{Materials and Methods}

\section{Cell culture}

The ovarian cancer cell lines SKOV3 and OVCAR3 were obtained from ATCC and cultured in Dulbecco's Modified Eagle Medium (DMEM) supplemented with 10\% FBS (Hyclone; Logan, UT), 100 U/ $\mathrm{ml}$ penicillin, and $100 \mu \mathrm{g} / \mathrm{ml}$ streptomycin (Invitrogen; Carlsbad, CA). HEK293 FT cells were cultured in DMEM media with 10\% FBS, $100 \mathrm{U} /$ $\mathrm{ml}$ penicillin, $100 \mu \mathrm{g} / \mathrm{ml}$ streptomycin, $1 \%$ glutamine, $1 \%$ nonessential amino acid, and $1 \mu \mathrm{g} / \mathrm{ml}$ geneticin.

\section{Lentiviral vector production}

miR-203 and EGFP lentiviral vectors were purchased from Applied Biological Materials Inc. (Richmond, Canada). Lentiviral shRNA vector (TRCN0000015388) for Snai2 knockdown was purchased from Dharmacon (Lafayette, CO). Lentiviral scramble shRNA(\#1864) was purchased from Addgene (Cambridge, MA). All lentiviral vectors were packaged in HEK293FT cells and produced as described previously [32]. miR-203 expressing, EGFP control and Snai2 shRNA cell lines were established by transducing SKOV 3 and OVCAR cells with purified virus, and stable pools of cells selected with $5 \mu \mathrm{g} / \mathrm{ml}$ puromycin.

\section{Cell colony formation assay}

SKOV3 and OVCAR3 cells transduced with miR-203 or EGFP control lentiviruses (200 cells/well) were plated in triplicate into 6-well plates and then stained with $0.1 \%$ Crystal Violet following a two-week culture, and cell colonies were counted as described previously [33].

\section{MTT assay}

The Cell Proliferation was examined using MTT Assay kit purchased from ATCC (Manassas, VA) following manufacturer's instructions. Ovarian cancer cells were plated (8,000 cells/well) in 96well plates. At different time points, $10 \mu \mathrm{l}$ of MTT reagent $(10 \mathrm{mg} /$ $\mathrm{mL}$ ) was added to each well and incubated for $\sim 4 \mathrm{~h}$. The reaction was terminated by adding $100 \mu \mathrm{l}$ lysis reagent and incubated at $22^{\circ} \mathrm{C}$ in the dark for $2 \mathrm{~h}$. The absorbance was measured at $570 \mathrm{~nm}$ wavelength on a Bio-Rad plate reader.

\section{Cell migration assay}

The transwell migration assay was performed using a modified chamber system from BD Falcon $^{\text {txi }}$ (San Jose, CA). These chambers were inserted into a 24 -well plate with $3 \times 10^{4}$ of cells in $300 \mu \mathrm{l}$ serumfree DMEM added to the upper chamber. DMEM (supplemented with $10 \%$ of FBS) was added into the lower chamber of each well as a chemoattractant, and cells were incubated for $24 \mathrm{~h}$. The medium and non-migrated cells in the upper chamber were removed, whereas the migrated cells on the lower side of the membranes were fixed with methanol and stained with $0.1 \%$ Crystal Violet. Pictures were taken at 10X magnification. Transmigrated cells in at least three different fields were counted.

\section{Cell invasion assay}

SKOV3 and OVCAR3 cells $\left(5 \times 10^{5}\right.$ per well $)$ transduced with miR203 and EGFP lentiviral vectors were seeded in serum-free DMEM onto inserts precoated with Matrigel (BD BioCoat ${ }^{\mathrm{TM}}, \mathrm{BD}$ BioSciences, San Jose, CA). DMEM supplemented with $10 \%$ FBS was added to the bottom chamber and $24 \mathrm{~h}$ later, the transwell inserts were stained using $4 \mu \mathrm{g} / \mathrm{ml}$ of Calcein AM (Life Technologies; Grand Island, NY) at $37^{\circ} \mathrm{C}$ for $1 \mathrm{~h}$. The fluorescence intensity was measured using the BioTek Synergy ${ }^{\mathrm{TM}}$ plate reader (Winooski, VT) at excitation and emission wavelengths of $485 \mathrm{~nm}$ and $528 \mathrm{~nm}$, respectively. The invasion rate was calculated by the fluorescence of invading cells in miR-203 expressing cells/control cells x100.

\section{Real-time RT-PCR}

Total RNA was extracted from ovarian cancer cells using Trizol or from 3-5 $(\mu \mathrm{m})$ curls cut from paraffin-embedded (FFPE) blocks of fully de-identified ovarian patient biopsy specimens (UTHSC Tissue Services Core), using the RecoverAll ${ }^{\mathrm{TM}}$ Total Nucleic Acid Isolation Kit (Ambion Inc.). Since the identities of the individuals providing the specimens were not readily ascertainable by the investigators, the UTHSC IRB determined that the regulations do not apply to the use of specimens in this study. In addition, because the regulations are not applicable to this research study, informed consent was waived by the UTHSC IRB when it reviewed the study. Total RNA was used to perform RT-PCR or polyA tailing real-time RT-PCR. The miRNA expression level was normalized against the expression of non-coding RNA U6 as described previously [32].

\section{Immunofluorescence staining}

Ovarian cancer cells were fixed for $10 \mathrm{~min}$ using $4 \%$ paraformaldehyde and washed three times with $0.1 \%$ Tween 20 in PBS (PBST). Sections from human and mouse tumors were antigenretrieved and incubated with blocking buffer (5\% normal goat serum, 3\% bovine serum albumin, and $0.1 \%$ Triton-X 100 in PBS) for $1 \mathrm{~h}$. Primary antibodies to E-cadherin, Snai2, vimentin (1:200; Cell Signaling; Danvers, MA), and PCNA (1:200; Santa Cruz) were incubated with the cells overnight. After three rinsing for 5 min with PBST, Alexa 594 conjugated goat anti-rabbit antibodies were applied (1:200 dilution; Life Technologies) for $1 \mathrm{~h}$ at room temperature. Cell nuclei were counterstained with DAPI (Vector Laboratories, Inc.; Burlingame, CA). Images were taken using a Nikon Ti inverted fluorescence microscope.

\section{Western blot}

Ovarian cancer cells were collected in RIPA buffer (Thermo Scientific; Rockford, IL) containing 1\% Halt Proteinase Inhibitor Cocktail (Thermo Scientific). An equal amount of protein $(40 \mu \mathrm{g} /$ lane) was loaded onto $10 \%$ SDS-PAGE gels and transferred onto nitrocellulose membranes. The membranes were blocked with $5 \%$ nonfat milk for $1 \mathrm{~h}$ and incubated with primary antibodies (1:1000 dilution) against glyceraldehyde 3-phosphate dehydrogenase (GAPDH; Sigma; St. Louis, MO), vimentin, E-cadherin, or Snai2 (Cell Signaling). 
Citation: Zhao G, Guo Y, Chen Z, Yang Y, Yang C, et al. (2015) miR-203 Functions as a Tumor Suppressor by Inhibiting Epithelial to Mesenchymal Transition in Ovarian Cancer. J Cancer Sci Ther 7: 034-043. doi:10.4172/1948-5956.1000322

\section{Mouse xenograft model}

Animal experiments were performed in accordance with a study protocol approved by the Institutional Animal Care and Use Committee of the University of Tennessee Health Science Center. SKOV3 and OVCAR3 cells $\left(1 \times 10^{6}\right)$ labeled with a lentiviral vector expressing luciferase (pLenti-UBC-Luc2-T2A-mKate;) were injected subcutaneously into two-month-old immunodeficient NOD.CgPrkdc ${ }^{\text {scid }} \mathrm{Il} 2 \mathrm{rg}^{\text {tm1Wjl }} / \mathrm{SzJ}$ (NSG) female mice. Six mice were used for each group. Tumor initiation and progression were monitored twice a week using a Xenogen imaging system. Tumor sizes were measured using a handheld caliper and tumor volume was calculated using the formula: $\left(\right.$ length $\times$ width $\left.^{2}\right) / 2$ [34]. All mice were sacrificed at two months following cell inoculation; the tumors were weighed and processed for $\mathrm{H} \& \mathrm{E}$ and immunostaining.

\section{TCGA database query}

To examine the association of the expression of miR-203 and its target gene Snai2 with clinical characteristics of human ovarian cancer patients, we queried the TCGA database (https://tcga-data.nci.nih.gov/ tcga/tcgaHome2.jsp). The data set was filtered for samples on miR-
203, Snai2, and clinical data. Statistical analyses were performed using Graphpad Prism software package.

\section{Statistical analysis}

Significant differences were determined using Student's $t$-test from at least two independent experiments performed in triplicate and presented as means \pm S.D. $p<0.05$ was considered significant.

\section{Results}

miR-203 expression correlates with a long-term survival in ovarian cancer patients and is downregulated in ovarian cancer

To determine whether miR-203 is associated with the clinical outcome of ovarian cancer patients, we analyzed miR-203 expression in top 10\% (33 cases) and lower 10\% (33 cases) based on survival of ovarian cancer patients in the TCGA database. We found that miR203 expression is significantly higher in the top $10 \%$ of surviving patients when compared to the lower $10 \%$ of surviving patients (Figure $1 \mathrm{~A} ; p=0.017)$. In addition, we also detected miR-203 expression in RNA extracted from FFPE tissue blocks of 16 human serous ovarian

\section{A}

miR-203 expression in ovarian tumors (TCGA)
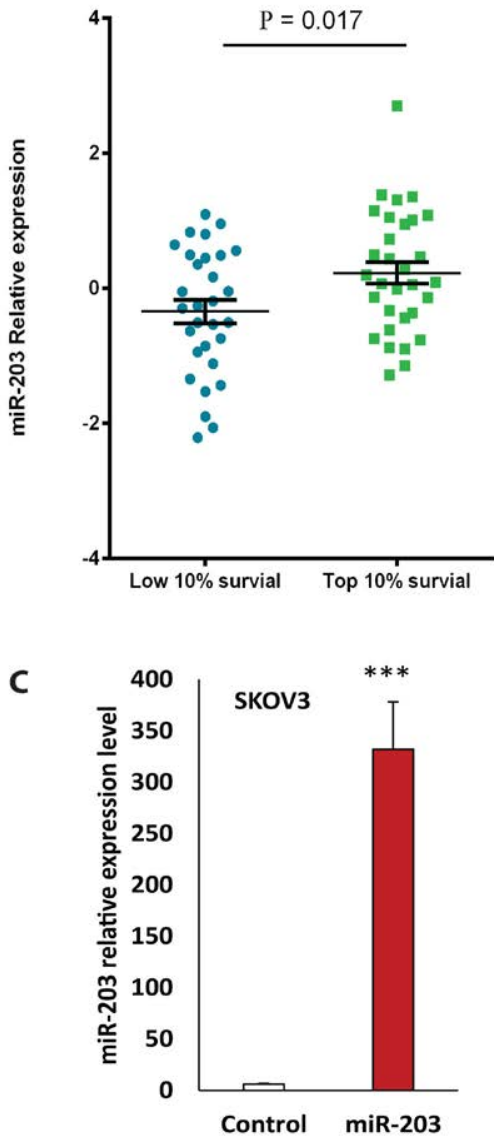

B

miR-203 expression in ovarian tumors (UT)
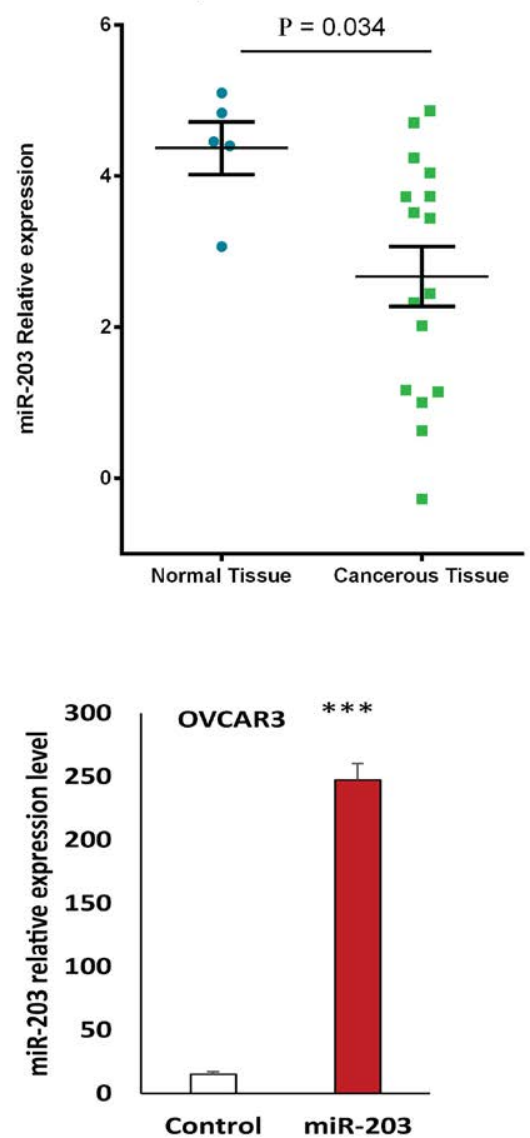

Figure 1: $\mathrm{miR-203}$ is associated with long-term survival of ovarian cancer patients and is downregulated in ovarian serous carcinoma. A. miR-203 expression in the top and lower $10 \%$ surviving patients with ovarian cancer was analyzed from the TCGA database. Significant differences was analyzed using Student's T test. * $p=0.017)$. B. miR-203 is downregulated in ovarian serous carcinoma compared to normal control ovaries $(p=0.034)$. C. miR-203 expression in SKOV3 and OVCAR3 cells transduced with miR-203 lentiviral and EGFP control vectors was determined by polyA tailing real-time RT-PCR and normalized to expression of the non-coding RNA U6. 
Citation: Zhao G, Guo Y, Chen Z, Yang Y, Yang C, et al. (2015) miR-203 Functions as a Tumor Suppressor by Inhibiting Epithelial to Mesenchymal Transition in Ovarian Cancer. J Cancer Sci Ther 7: 034-043. doi:10.4172/1948-5956.1000322

carcinoma and in 5 adjacent normal ovary specimens. We found that miR-203 was significantly downregulated in human ovarian carcinoma compared to normal ovary controls (Figure $1 \mathrm{~B} ; p=0.034$ ). Taken together, these findings demonstrate that miR-203 expression is positively correlated with the survival of ovarian cancer patients.

miR-203 inhibits cell proliferation, survival, migration, and invasion in ovarian carcinoma cells

Although miR-203 has been reported to function as a tumor suppressor [35-37], its role in ovarian cancer has not yet been elucidated. To address the role of miR-203 in ovarian cancer, we overexpressed miR-203 in SKOV3 and OVCAR3 cells using a lentiviral vector by 55 -fold and 22-fold, respectively, compared to EGFP control vectortransduced cells (Figure 1C). We then determined whether miR-203 overexpression affects the proliferation of ovarian cancer cells. The cell proliferation rates of empty vector- and miR-203-transduced SKOV3 and OVCAR3 cells were compared over a four-day culture period using the MTT assay. We found that proliferation of miR-203 transduced SKOV3 and OVCAR3 cells was significantly reduced when compared to empty-vector transduced cells (Figure 2A). To examine whether miR-203 affects cell survival, we performed colony formation assays in miR-203-expressing SKOV3 and OVCAR3 cells. Cell colonies were significantly reduced in miR-203-expressing SKOV3 and OVCAR3 cells compared to control cells (Figure 2B). We also studied the effect of miR-203 on the migration and invasion of ovarian cancer cells by using transwell plates coated with or without Matrigel to quantify invasion and migration, respectively. As shown in Figure 2C and D, migration and invasion were significantly reduced in miR-203-expressing SKOV3 and OVCAR3 cells when compared to control cells. These data suggest that miR-203 overexpression inhibits ovarian cancer cell proliferation, survival, migration and invasion.
A.
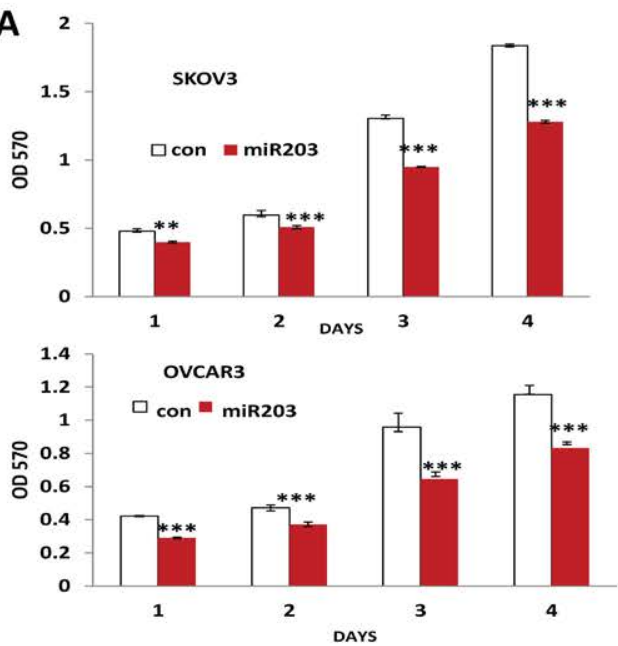

B
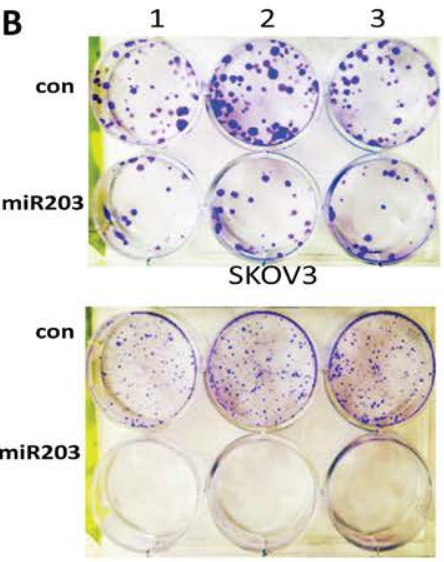

OVCAR3
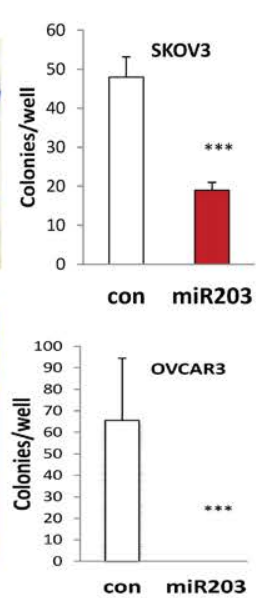

C
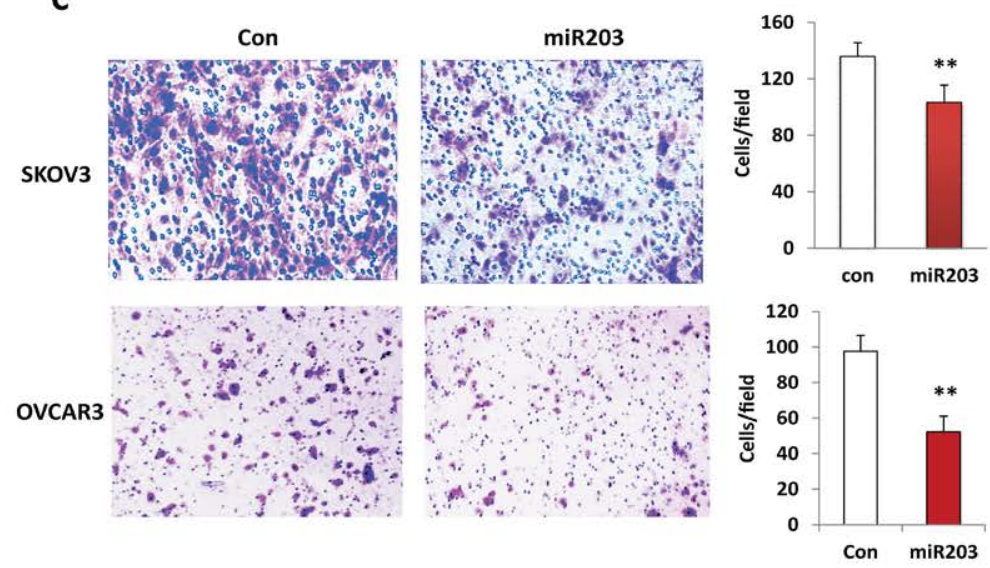

Figure 2: miR-203 inhibits cell proliferation, survival, migration and invasion in ovarian cancer cells. A. Cell proliferation in SKOV3 and OVCAR3 cells transduced with either lentiviral miR-203 or EGFP controls was examined using MTT assay. Overexpression of miR-203 significantly inhibits cell proliferation in both SKOV3 and OVCAR3 cells compared to that in control cells as analyzed by Student's T test ( $\left.{ }^{*} p<0.05\right)$. B. SKOV3 and OVCAR cells transduced with lentiviral miR-203 or EGFP control vectors were seeded into 6 -well plates and cultured for $14 \mathrm{~d}$. Cell colonies were counted following Crystal Violet staining. The number of colonies in miR-203-overexpressing cells was significantly reduced compared to that in control cells analyzed by Student's T test $\left({ }^{* *} p<0.001\right)$. C. Transwell migration assays were performed in miR-203-expressing and control SKOV3 and OVCAR3 cells. Transmigrated cells were stained with Crystal Violet and counted. Overexpression of miR-203 significantly reduced cell migration in both SKOV3 and OVCAR3 cells compared with control cells $\left({ }^{* * *} p<0.001\right)$. D. Invasion assays were performed using Matrigel-coated transwell plates for miR-203-expressing and control SKOV3 and OVCAR3 cells. The invasion rate was calculated based on the invasion of miR-203overexpressing SKOV3 and OVCAR3 cells relative to control cells $\left({ }^{* *} p<0.01\right)$. Data were collected from three separate experiments and analyzed using Student $t$-tests. 
Citation: Zhao G, Guo Y, Chen Z, Yang Y, Yang C, et al. (2015) miR-203 Functions as a Tumor Suppressor by Inhibiting Epithelial to Mesenchymal Transition in Ovarian Cancer. J Cancer Sci Ther 7: 034-043. doi:10.4172/1948-5956.1000322

\section{miR-203 inhibits spontaneous EMT in ovarian cancer cells}

MiRNAs function by downregulating the expression of target genes. Previous studies showed that miR-203 targets Snai2 in prostate and breast cancer [37,38]. A putative miR-203 binding sequence is present at positions 351 to 358 in the 3 ' untranslated region of the Snai2 gene (Figure $3 \mathrm{~A})$. Snai2 is a mesenchymal cell marker in various human cancers and functions as a key regulator of EMT [39-41]. To examine whether miR-203 expression regulates EMT in ovarian cancer cells, we examined the expression of Snai2, the epithelial cell marker E-cadherin and the mesenchymal marker vimentin in miR-203expressing SKOV3 and OVCAR3 cells. The expression of E-cadherin was significantly upregulated, whereas vimentin and Snai2 were significantly downregulated in both miR-203-expressing SKOV3 (Figure 3B) and OVCAR3 (Figure 3C) cells when compared to control cells.

\section{miR-203 inhibits tumor growth in vivo}

To determine the effect of miR-203 on tumor growth in vivo, miR203-expressing SKOV3 and OVCAR3 cells transduced with a lentiviral luciferase vector were injected subcutaneously into immunodeficient NSG female mice. We found that tumors were significantly smaller in mice xenografted with miR-203-expressing SKOV3 and OVCAR3 cells compared to control cells as shown by Xenogen live animal imaging (Figure 4A and B). Tumor volume and weight were also significantly reduced in mice injected with miR-203-expressing SKOV3 and OVCAR3 cells compared to controls (Figure 4C and D). To examine the expression of EMT marker genes and cell proliferation in tumor xenografts of control or miR-203-expressing SKOV3 cells, we immunostained tumor sections with antibodies to Snai2, E-cadherin and vimentin, as well as the cell proliferation marker PCNA. Snai2 (Figure 5A), vimentin (Figure 5B) and PCNA (Figure 5C) were downregulated, whereas E-cadherin (Figure 5D) was upregulated in tumors xenografted with miR-203-expressing SKOV3 cells compared to controls, suggesting that miR-203 inhibits EMT and tumor growth in vivo and functions as a tumor suppressor in ovarian cancer.

\section{Silencing Snai2 expression mimics miR-203-mediated function in ovarian cancer cells}

Because Snai2 is a miR-203 target gene, we hypothesized that silencing Snai2 may mimic some of miR-203 function in ovarian cancer cells. To test this hypothesis, we transduced SKOV3 and OVCAR3 cells with Snai2 lentiviral shRNA (Snai2KD) and scrambled control vectors (SC). We found that cell survival (Figure 6A), migration (Figure 6B) and invasion (Figure 6C) were significantly inhibited in Snai2 knockdown SKOV3 and OVCAR3 cells compared to SC controls. We

A

Position 351-358 of Snai2 3' UTR 5' ACAUUGCUGCCAAAUCAUUUCAA

hsa-miR-203

B

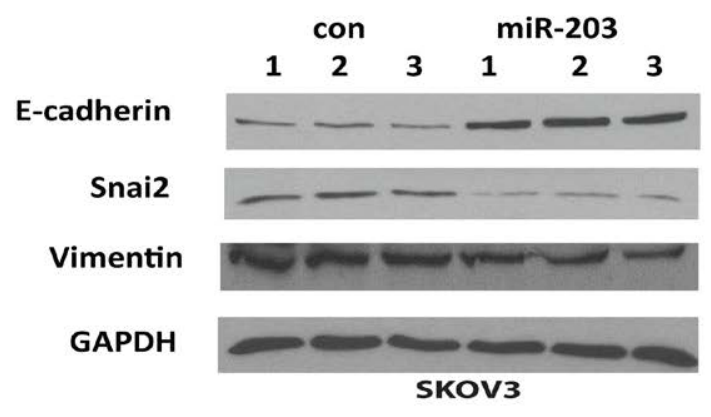

C

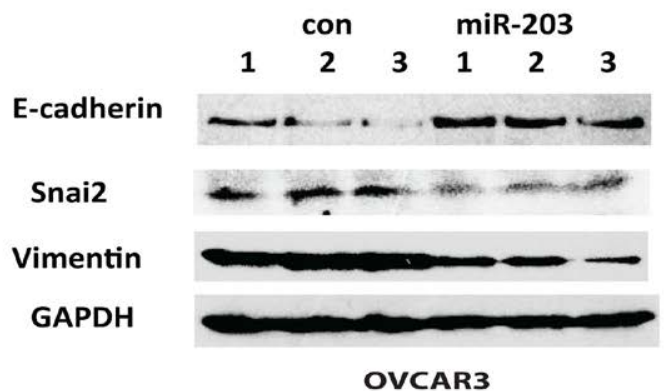

Figure 3: miR-203 inhibits EMT and suppresses TGF $\beta$-induced EMT in ovarian cancer cells. A. miR-203 binding sites in the 3' UTR of human Snai2 gene. B, C. EMT marker genes Snai2, vimentin, and E-cadherin were examined in miR-203-expressing and control SKOV3 and OVCAR3 cells by Western blot. One representative Western blot was presented from three independent experiments. 
Citation: Zhao G, Guo Y, Chen Z, Yang Y, Yang C, et al. (2015) miR-203 Functions as a Tumor Suppressor by Inhibiting Epithelial to Mesenchymal Transition in Ovarian Cancer. J Cancer Sci Ther 7: 034-043. doi:10.4172/1948-5956.1000322

A

A

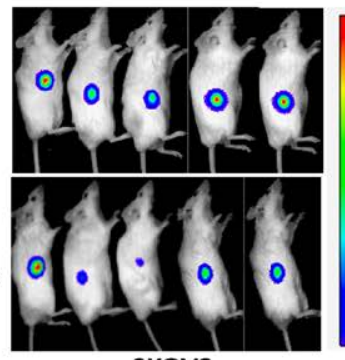

SKOV3

B
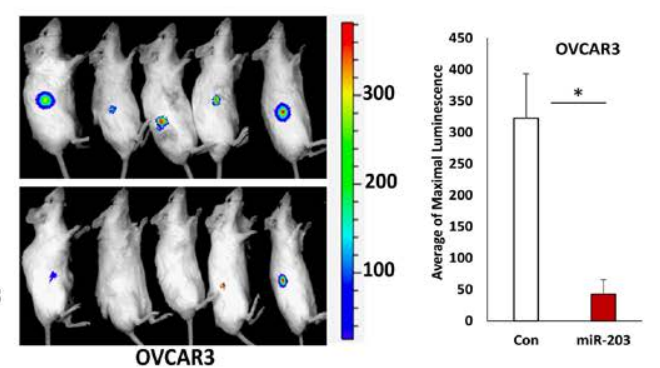

\section{C}
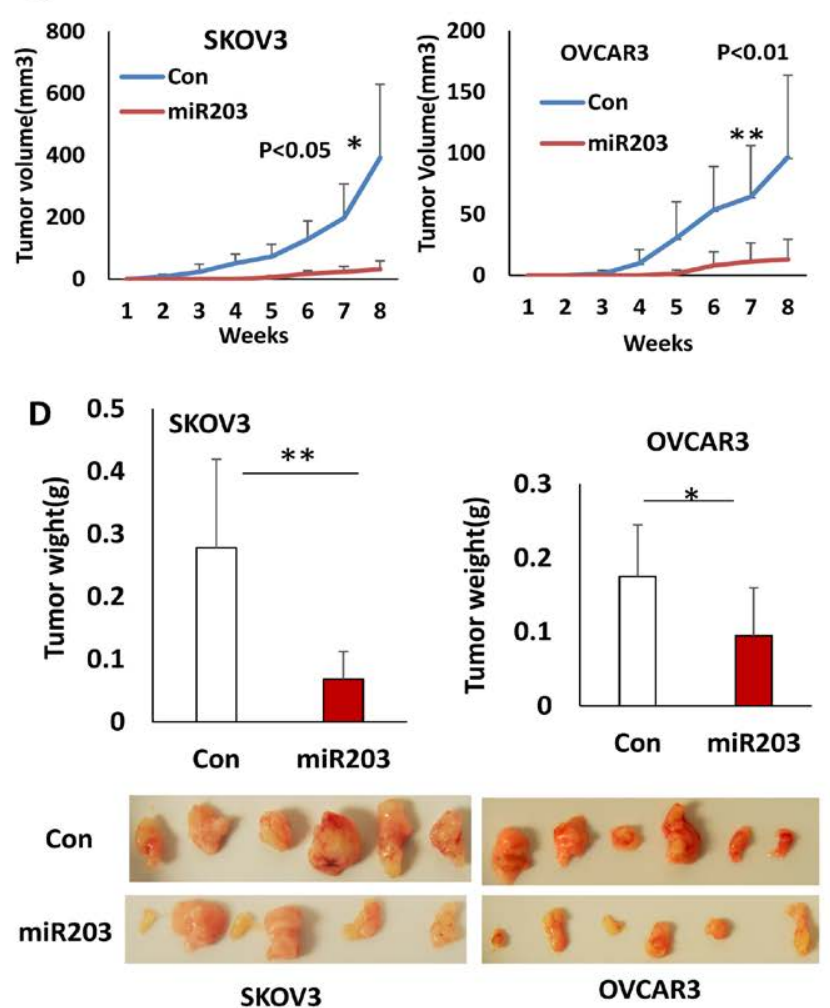

Figure 4: miR-203 inhibits tumor growth in a xenograft mouse model. A, B. miR-203-expressing SKOV3, OVCAR3 and control cells were subcutaneously xenografted into immunodeficient NSG female mice and imaged at two weeks after injection by Xenogen live animal imaging. Tumor size was evaluated using the average maximal luminescence from 6 mice $\left({ }^{*} p<0.05\right)$. C. Tumor volumes in NSG mice xenografted with miR-203-expressing SKOV3 and OVCAR3 cells were measured using a caliper. $\left({ }^{*} p<0.05 ;{ }^{* *} p<0.01\right)$. D. Tumors from NSG mice xenografted with miR-203-expressing SKOV3 and OVCAR3 cells were dissected and weighed $\left({ }^{*} p<0.05 ;{ }^{* *} p<0.01\right)$. Significance was analyzed using Student's T test.

A

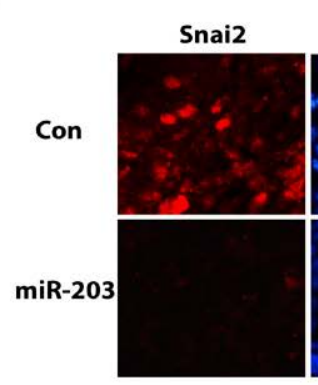

B

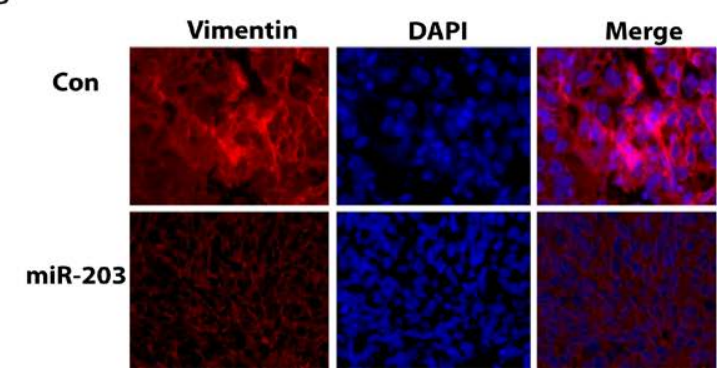

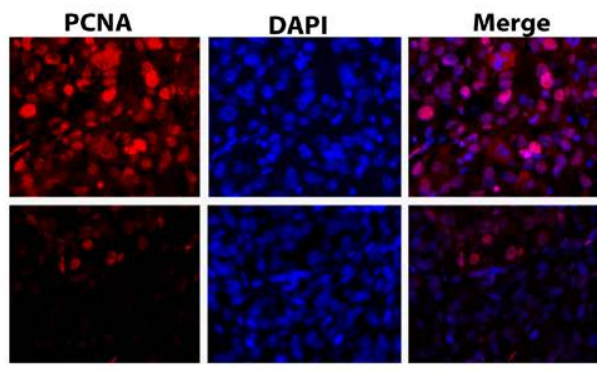

D

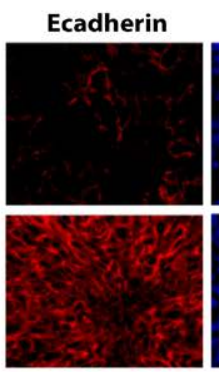

Merge

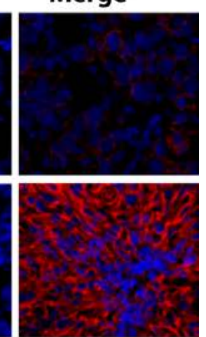

Figure 5: miR-203 inhibits EMT in mouse tumors xenografted with ovarian cancer cells. A, B, C, D. Sections of mouse tumor xenografted from miR-203expressing and control SKOV3 cells were immunostained with EMT marker genes, Snai2, vimentin and E-cadherin, as well as the cell proliferation maker gene PCNA. Representative images were shown from tumors collected from three different mice xenografted with miR-203 expressing and control SKOV3 cells. 
A

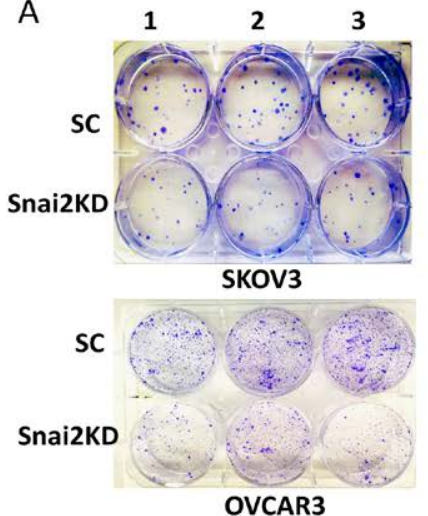

B

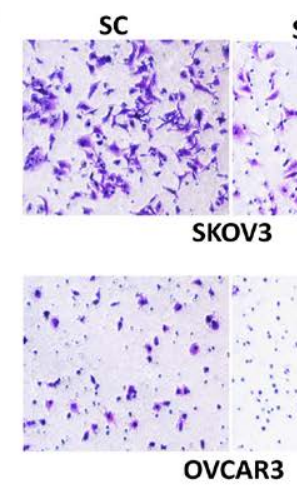

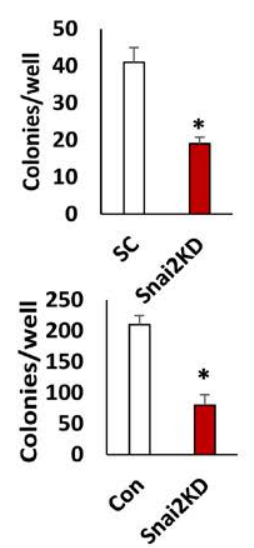

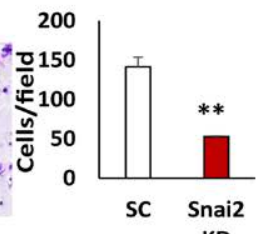

KD

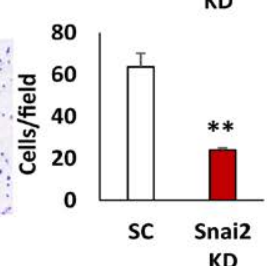

C
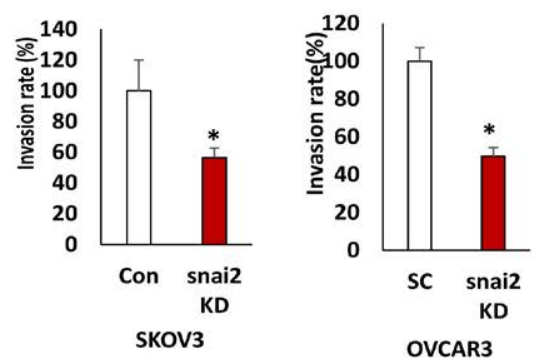

D

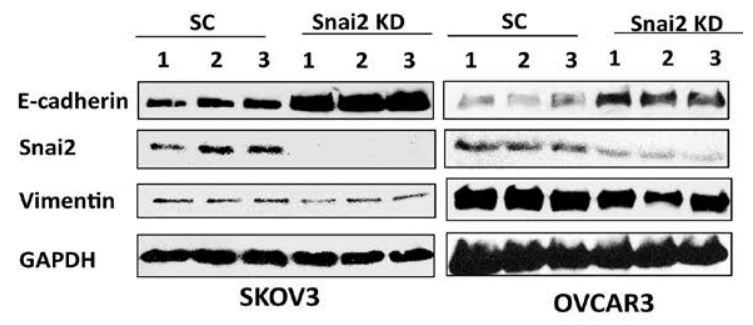

$\mathbf{E}$
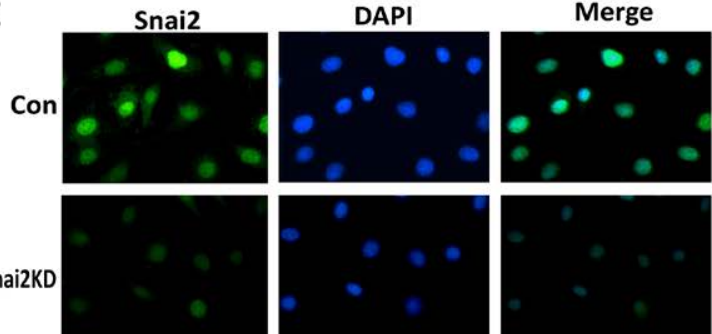

Figure 6: Silencing Snai2 mimics miR-203 function in ovarian cancer cells. A. Cell colony formation assays were performed using SKOV3 and OVCAR3 cells transduced with lentiviral Snai2 and SC shRNA. Cells were cultured for 2 weeks, and then stained with Crystal Violet. ${ }^{*} p<0.05$. B. Migrated SKOV3 and OVCAR3 cells transduced with lentiviral Snai2 and SC shRNA were stained using Crystal Violet and counted. ${ }^{* *} p<0.01$. C. Snai2 knockdown SKOV3, OVCAR3, and control cells were seeded in Matrigel-coated transwells and invaded cells were quantified by measuring the fluorescent intensity following calcein staining. ${ }^{*} p<0.05$. $\mathbf{D}$. EMT marker genes in Snai2 knockdown SKOV3 and OVCAR3 cells were determined using Western blot. One representative Western blot was shown from three independent experiments. E. Representative image of immunostaining on Snai2 expression in SKOV3 cells transduced with Snai2 and SC lentiviral shRNAs.

also examined EMT marker genes using Western blot and found that knockdown of Snai2 significantly reduced vimentin expression whereas E-cadherin expression was upregulated. This finding indicates that Snai2 knockdown suppresses EMT in ovarian cancer cells (Figure 6D). Snai2 expression in SKOV3 cells transduced with lentiviral shRNA and SC controls was evaluated by immunostaining with antibodies to Snai2. Snai2 downregulation in knockdown cells was verified in comparison to SC controls (Figure 6E).

\section{Snai2 is associated with short-term survival and is upregulated in human ovarian cancer specimens}

To determine the relationship of Snai2 expression with the clinical outcome of ovarian cancer patients, we analyzed Snai2 expression in the top and lower $10 \%$ surviving ovarian carcinoma patients present in the TCGA database. Snai2 expression was significantly higher in low $10 \%$ surviving patients compared to top $10 \%$ surviving patients (Figure 7A; $p=0.0202$ ). We also examined Snai2 expression in FFPE tissue blocks of 16 serous carcinomas and 5 normal ovarian tissues. Snai2 expression was significantly upregulated in serous carcinoma compared to normal ovarian tissue (Figure 7B). We also performed immunostaining on sections of human ovarian serous carcinoma specimens and normal ovarian tissue using antibodies to Snai2 and PCNA. We found high expression of Snai2 and PCNA expression in tumor tissue (Figure 7C) relative to normal controls (Figure 7D). The morphology for ovarian serous carcinoma (Figure 7E) and normal ovarian tissue (Figure 7F) were verified by $\mathrm{H} \& \mathrm{E}$ staining. Taken together, our results indicate that Snai2 is regulated by miR-203. Moreover, Snai2 expression is inversely correlated with miR-203 expression in human serous ovarian carcinoma and correlates with short-term survival of ovarian cancer patients.

\section{Discussion}

In this study, we showed that miR-203 inhibited cell proliferation, migration, invasion and tumor growth of ovarian cancer cells by targeting the transcriptional factor Snai2. miR-203 was highly expressed in both ovarian cancer cell lines. We do not believe these effects simply reflect the use of a viral vector to drive miR-203 overexpression, since similar results were obtained in two different ovarian cancer cell types. Moreover, the findings in this study are similar to what has been reported in prostate [29] and breast cancer [30]. So off-target effects are relatively low. However, in future studies we will consider using a doxycycline inducible system to control miR-203 expression in our next study to answer this question from a different approach. We also demonstrated that miR-203 is capable of inhibiting EMT in ovarian cancer cells (Figure 7G). miR-203 expression is downregulated and inversely correlated with Snai2 expression in human ovarian carcinoma as compared to normal controls. High miR-203 and Snai2 expression correlated with the top and lower $10 \%$ surviving patients 
Citation: Zhao G, Guo Y, Chen Z, Yang Y, Yang C, et al. (2015) miR-203 Functions as a Tumor Suppressor by Inhibiting Epithelial to Mesenchymal Transition in Ovarian Cancer. J Cancer Sci Ther 7: 034-043. doi:10.4172/1948-5956.1000322

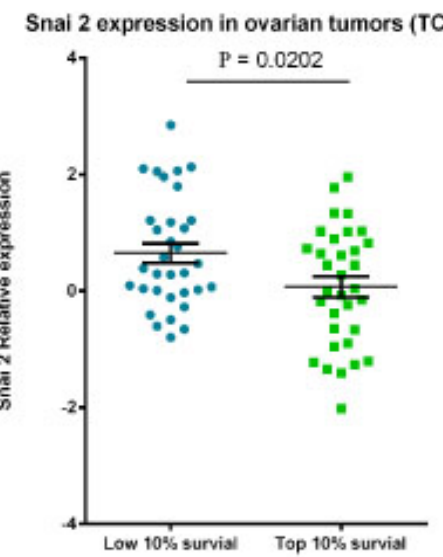

B

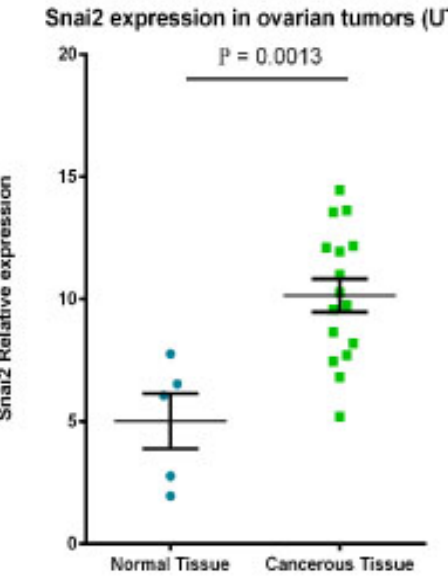

C
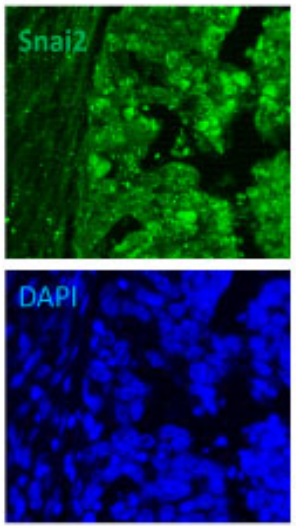

D
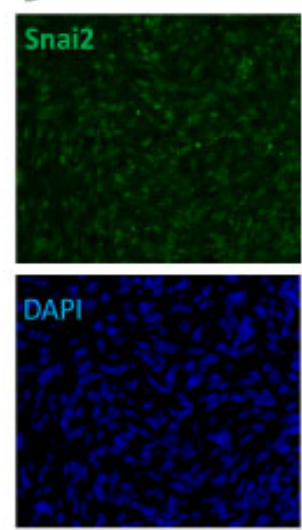
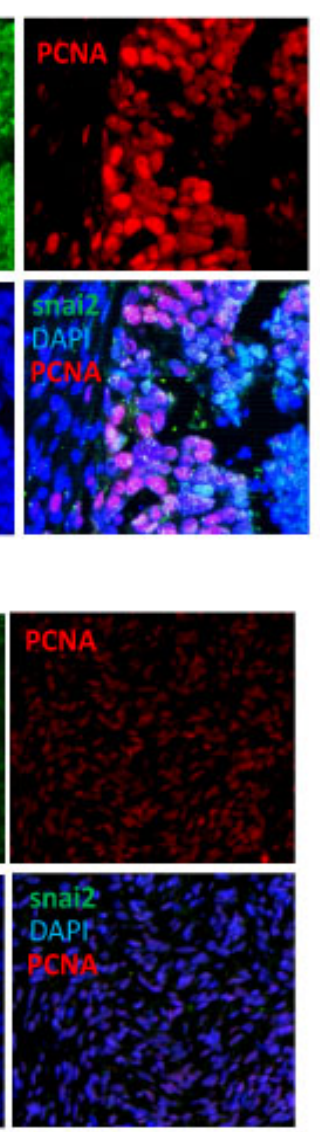

$\mathrm{E}$
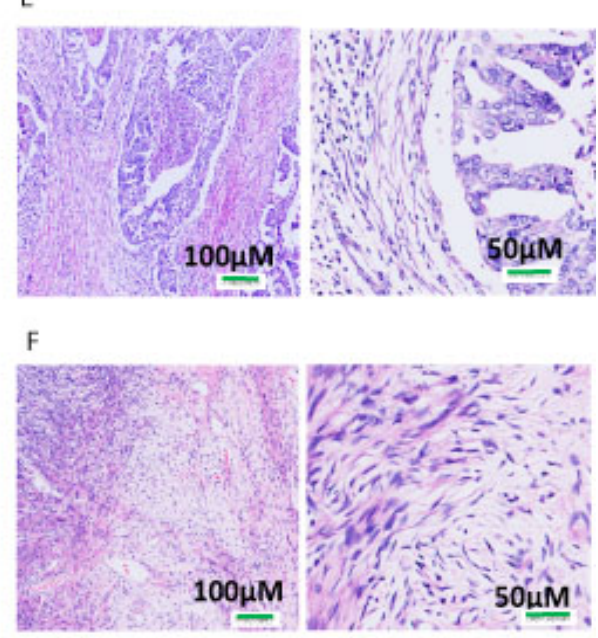

G

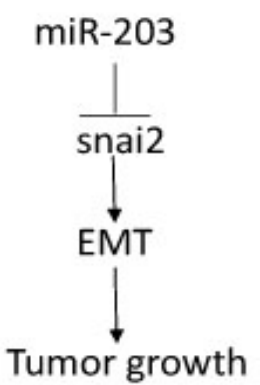

Figure 7: Snai2 is associated with short-term survival of ovarian cancer patients and is upregulated in ovarian serous carcinoma. A, Snai2 expression in top and lower $10 \%$ of surviving patients with ovarian cancer in the TCGA database $(p=0.0202)$. B. Snai2 expression was examined in 16 human serious carcinoma and 5 normal ovarian tissue specimens using real-time RT-PCR $(p=0.0013)$. C, D. One representative image of Snai2 and PCNA expression was shown by immunofluorescence staining of sections of six different human serous ovarian carcinomas (C) and three normal ovarian tissue specimens (D). E, F. Representative H\&E-stained sections of six serous carcinomas (E) and three normal ovarian tissue specimens (F) with low and high magnification. G. miR-203 functions as a tumor suppressor in ovarian cancer by downregulating Snai2 as described in the schematic diagram.

with ovarian cancer, respectively. Our findings indicate that miR-203 is a potential prognostic biomarker in ovarian cancer patients. Further studies are warranted to define the relationship of miR-203 expression with other forms of ovarian cancer, including serous, endometrioid, clear cell, and mucinous. It will also be interesting to examine whether miR-203 expression correlates with the different stages and grades of ovarian cancer. Although the majority of studies showed that miR203 is downregulated in several human cancers [22,42-44], one study reported miR-203 upregulation in ovarian cancer as compared to normal ovary controls [31]. It is possible that different expression levels of miR-203 may reflect sample variation in collection from different stages in tumor development. Clearly, more studies are needed to characterize miR-203 expression in ovarian cancers. Although the sample size used in our study is relatively small and represents only ovarian serous carcinoma specimens, it is important to note that more than $70 \%$ of ovarian cancer patients are diagnosed with the serous type of carcinoma [45].

We demonstrated that miR-203 inhibits EMT in ovarian cancer cells. TGF $\beta$ has been shown to induce EMT in ovarian cancer cells [46]. However, it is not clear how miR-203 regulates the TGF $\beta$ signaling pathway. Previous studies showed that miR-181a promotes TGF $\beta$ - induced EMT by targeting Smad7, thus activating the TGF $\beta$ signaling pathway and promoting tumorigenesis in ovarian cancer. Activation of the TGF $\beta$ pathway is correlated with poor survival in ovarian cancer [27]. Snai2 is activated by TGF $\beta$ and is a downstream target of the TGF $\beta$ signaling pathway in prostate cancer and breast cancer $[42,47]$. Snai2 expression activates TGF $\beta$ signaling by upregulating TGF $\beta$ receptor2 (TGFR2) through promoting histone $\mathrm{H} 3 \mathrm{~K} 9$ acetylation in breast cancer [40]. However, whether miR-203 inhibits EMT by counteracting TGF $\beta$ induced EMT in ovarian cancer requires further investigation. In addition to miR-203 directly targeting the EMT marker gene Snai2, several other miRNAs also regulate EMT marker genes in various human cancers. miR-506 suppresses EMT by targeting Snai2, vimentin and $\mathrm{N}$-cadherin in ovarian cancer [12,25]. miR-101, miR-150, and miR-200c inhibit EMT by targeting zinc finger E-box binding homeobox 1 factors ZEB1 and ZEB2 in colon, pancreatic, and ovarian cancers $[11,48,49]$. These studies demonstrate that miRNAs are important regulators of EMT by directly downregulating EMT marker genes. Silencing Snai2 expression in SKOV3 and OVCAR3 cells by lentiviral shRNA knockdown, we show that Snai2 down regulation mimics miR-203 function in inhibiting cell proliferation, migration, and invasion. Moreover, Snai2 knockdown upregulates E-cadherin 
and downregulates vimentin in both cell lines. Therefore, the miR$203 /$ Snai2 2 axis plays a key role in inhibiting ovarian cancer growth by regulating EMT.

We also found that miR-203 sensitizes ovarian cancer SKOV3 and OVCAR2 cells to cisplatin treatment (data not shown). Therefore, miR203 increases the sensitivity of ovarian cancer cells to chemotherapy at least partially through inhibiting EMT. The miRNAs/EMT axis in ovarian cancer metastasis has been studied by using orthotopic ovarian cancer mouse models. Overexpression of miR-506 or knockdown of miR-181a inhibited EMT in ovarian cancer cells and also suppressed the metastatic progression $[25,27]$. We showed that miR-203 inhibited tumor growth using a subcutaneous xenograft mouse model. Immunostaining of tumor sections from NSG mice xenografted with miR-203 and control SKOV3 cells with antibodies against EMT marker genes confirmed that miR-203 inhibits EMT in vivo. However, it is still unclear how miR-203 regulates EMT/MET interconversion during the metastatic progression from primary to distant tumor sites via spheroids in ascites. Therefore, further studies are needed to define how miR-203 plays a role in metastasis using orthotopic ovarian cancer mouse models.

In summary, this is the first report showing that miR-203 functions as a tumor suppressor by inhibiting cell proliferation, migration, and invasion in ovarian cancer cells as well as tumor growth in vivo through downregulating Snai2 and inhibiting EMT.

\section{Acknowledgments}

This project was partially supported by grants from the National Institute of Health CA092160 (G.T), CA133322 (L.M.P.), the Department of Defense W81XWH-11-1-0533 (L.M.P.), the West Cancer Center (J.Y.), and the Chinese Government (112102310110, 122102310553 to Y.G., 2011DFA33290 to W.G.) We thank Dr. Anand Kulkarni, who reviewed the tissue blocks of human ovarian carcinoma.

\section{References}

1. Siegel R, Naishadham D, Jemal A (2012) Cancer statistics, 2012. CA Cancer $\mathrm{J}$ Clin 62: 10-29.

2. Yue $P$, Zhang $X$, Paladino D, Sengupta B, Ahmad S, et al. (2012) Hyperactive EGF receptor, Jaks and Stat3 signaling promote enhanced colony-forming ability, motility and migration of cisplatin-resistant ovarian cancer cells. Oncogene 31: 2309-2322.

3. Chou JL, Su HY, Chen LY, Liao YP, Hartman-Frey C, et al. (2010) Promoter hypermethylation of FBXO, a novel TGF-beta/SMAD4 target gene and tumo suppressor, is associated with poor prognosis in human ovarian cancer. Lab Invest 90: 414-425.

4. Cañadas I, Rojo F, Taus Á, Arpí O, Arumí-Uría M, et al. (2014) Targeting epithelial-to-mesenchymal transition with Met inhibitors reverts chemoresistance in small cell lung cancer. Clin Cancer Res 20: 938-950.

5. Kajiyama H, Shibata K, Terauchi M, Yamashita M, Ino K, et al. (2007) Chemoresistance to paclitaxel induces epithelial-mesenchymal transition and enhances metastatic potential for epithelial ovarian carcinoma cells. Int J Oncol 31: $277-283$.

6. Güngör C, Zander H, Effenberger KE, Vashist YK, Kalinina T, et al. (2011) Notch signaling activated by replication stress-induced expression of midkine drives epithelial-mesenchymal transition and chemoresistance in pancreatic cancer. Cancer Res 71: 5009-5019.

7. Glackin CA (2014) Targeting the Twist and Wnt signaling pathways in metastatic breast cancer. Maturitas 79: 48-51.

8. Korsching E, Packeisen J, Liedtke C, Hungermann D, Wülfing $P$, et al (2005) The origin of vimentin expression in invasive breast cancer: epithelialmesenchymal transition, myoepithelial histogenesis or histogenesis from progenitor cells with bilinear differentiation potential? J Pathol 206: 451-457.

9. Tiwari N, Meyer-Schaller N, Arnold P, Antoniadis H, Pachkov M, et al. (2013) KIf4 is a transcriptional regulator of genes critical for EMT, including Jnk1 (Mapk8). PLoS One 8: e57329.
10. Pinho AV, Rooman I, Real FX (2011) p53-dependent regulation of growth, epithelial-mesenchymal transition and stemness in normal pancreatic epithelia cells. Cell Cycle 10: 1312-1321.

11. Wellner U, Schubert J, Burk UC, Schmalhofer O, Zhu F, et al. (2009) The EMTactivator ZEB1 promotes tumorigenicity by repressing stemness-inhibiting microRNAs. Nat Cell Biol 11: 1487-1495.

12. Luo X, Dong Z, Chen Y, Yang L, Lai D (2013) Enrichment of ovarian cancer stem-like cells is associated with epithelial to mesenchymal transition through an miRNA-activated AKT pathway. Cell Prolif 46: 436-446.

13. Lili LN, Matyunina LV, Walker LD, Wells SL, Benigno BB, et al. (2013) Molecular profiling supports the role of epithelial-to-mesenchymal transition (EMT) in ovarian cancer metastasis. J Ovarian Res 6: 49.

14. Ahmed N, Abubaker K, Findlay J, Quinn M (2010) Epithelial mesenchyma transition and cancer stem cell-like phenotypes facilitate chemoresistance in recurrent ovarian cancer. Curr Cancer Drug Targets 10: 268-278.

15. Wang N, Dong CR, Jiang R, Tang C, Yang L, et al. (2013) Overexpression of HIF-1alpha, metallothionein and SLUG is associated with high TNM stage and lymph node metastasis in papillary thyroid carcinoma. Int J Clin Exp Pathol 7 322-330.

16. Ahmed N, Thompson EW, Quinn MA (2007) Epithelial-mesenchymal interconversions in normal ovarian surface epithelium and ovarian carcinomas: an exception to the norm. J Cell Physiol 213: 581-588.

17. Helleman J, Smid M, Jansen MP, van der Burg ME, Berns EM (2010) Pathway analysis of gene lists associated with platinum-based chemotherapy resistance in ovarian cancer: the big picture. Gynecol Oncol 117: 170-176.

18. Yoshida S, Furukawa N, Haruta S, Tanase Y, Kanayama S, et al. (2009) Expression profiles of genes involved in poor prognosis of epithelial ovarian carcinoma: a review. Int J Gynecol Cancer 19: 992-997.

19. Miyamoto S, Nakanishi M, Rosenberg DW (2013) Suppression of colon carcinogenesis by targeting Notch signaling. Carcinogenesis 34: 2415-2423.

20. Liu YN, Abou-Kheir W, Yin JJ, Fang L, Hynes P, et al. (2012) Critical and reciprocal regulation of KLF4 and SLUG in transforming growth factor $\beta$-initiated prostate cancer epithelial-mesenchymal transition. Mol Cell Biol 32: 941-953.

21. Hoffmeyer K, Raggioli A, Rudloff S, Anton R, Hierholzer A, et al. (2012) Wnt// $\mathbf{I}^{2}$ catenin signaling regulates telomerase in stem cells and cancer cells. Science 336: $1549-1554$

22. Colomiere M, Ward AC, Riley C, Trenerry MK, Cameron-Smith D, et al. (2009) Cross talk of signals between EGFR and IL-6R through JAK2/STAT3 mediate epithelial-mesenchymal transition in ovarian carcinomas. $\mathrm{Br} \mathrm{J}$ Cancer 100 : 134-144.

23. Pon YL, Zhou HY, Cheung AN, Ngan HY, Wong AS (2008) p70 S6 kinase promotes epithelial to mesenchymal transition through snail induction in ovarian cancer cells. Cancer Res 68: 6524-6532.

24. Dong P, Kaneuchi M, Watari H, Hamada J, Sudo S, et al. (2011) MicroRNA-194 inhibits epithelial to mesenchymal transition of endometrial cancer cells by targeting oncogene BMI-1. Mol Cancer 10: 99

25. Sun Y, Hu L, Zheng H, Bagnoli M, Guo Y, et al. (2015) MiR-506 inhibits multiple targets in the epithelial-to-mesenchymal transition network and is associated with good prognosis in epithelial ovarian cancer. J Pathol 235: 25-36.

26. Hu X, Feng Y, Zhang D, Zhao SD, Hu Z, et al. (2014) A functional genomic approach identifies FAL1 as an oncogenic long noncoding RNA that associates with BMI1 and represses p21 expression in cancer. Cancer Cell 26: 344-357.

27. Parikh A, Lee C, Joseph P, Marchini S, Baccarini A, et al. (2014) microRNA181a has a critical role in ovarian cancer progression through the regulation of the epithelial-mesenchymal transition. Nat Commun 5: 2977.

28. Wang C, Zheng X, Shen C, Shi Y (2012) MicroRNA-203 suppresses cell proliferation and migration by targeting BIRC5 and LASP1 in human triplenegative breast cancer cells. J Exp Clin Cancer Res 31: 58.

29. Viticchiè G, Lena AM, Latina A, Formosa A, Gregersen LH, et al. (2011) MiR203 controls proliferation, migration and invasive potential of prostate cancer cell lines. Cell Cycle 10: 1121-1131.

30. Wang N, Liang H, Zhou Y, Wang C, Zhang S, et al. (2014) miR-203 suppresses the proliferation and migration and promotes the apoptosis of lung cancer cells by targeting SRC. PLoS One 9: e105570. 
Citation: Zhao G, Guo Y, Chen Z, Yang Y, Yang C, et al. (2015) miR-203 Functions as a Tumor Suppressor by Inhibiting Epithelial to Mesenchymal Transition in Ovarian Cancer. J Cancer Sci Ther 7: 034-043. doi:10.4172/1948-5956.1000322

31. Wang S, Zhao X, Wang J, Wen Y, Zhang L, et al. (2013) Upregulation of microRNA-203 is associated with advanced tumor progression and poor prognosis in epithelial ovarian cancer. Med Oncol 30: 681.

32. Yue J, Sheng Y, Ren A, Penmatsa S (2010) A miR-21 hairpin structure-based gene knockdown vector. Biochem Biophys Res Commun 394: 667-672.

33. Guo Y, Tian P, Yang C, Liang Z, Li M, et al. (2013) Silencing the DoubleStranded RNA Binding Protein DGCR8 Inhibits Ovarian Cancer Cell Proliferation, Migration, and Invasion. Pharm Res.

34. Jensen MM, Jørgensen JT, Binderup T, Kjaer A (2008) Tumor volume in subcutaneous mouse xenografts measured by microCT is more accurate and reproducible than determined by 18 F-FDG-microPET or external caliper. BMC Med Imaging 8: 16.

35. Miao L, Xiong X, Lin Y, Cheng Y, Lu J, et al. (2014) miR-203 inhibits tumor cell migration and invasion via caveolin-1 in pancreatic cancer cells. Oncol Lett 7 : 658-662.

36. Okumura T, Shimada Y, Moriyama M, Takei Y, Omura T, et al. (2014) MicroRNA-203 inhibits the progression of esophageal squamous cell carcinoma with restored epithelial tissue architecture in vivo. Int J Oncol 44: 1923-1932.

37. Qu Y, Li WC, Hellem MR, Rostad K, Popa M, et al. (2013) MiR-182 and miR203 induce mesenchymal to epithelial transition and self-sufficiency of growth signals via repressing SNAI2 in prostate cells. Int J Cancer 133: 544-555.

38. Zhang Z, Zhang B, Li W, Fu L, Fu L, et al. (2011) Epigenetic Silencing of miR203 Upregulates SNAI2 and Contributes to the Invasiveness of Malignant Breast Cancer Cells. Genes Cancer 2: 782-791.

39. Cates JM, Byrd RH, Fohn LE, Tatsas AD, Washington MK, et al. (2009) Epithelialmesenchymal transition markers in pancreatic ductal adenocarcinoma. Pancreas 38: e1-6.

40. Dhasarathy A, Phadke D, Mav D, Shah RR, Wade PA (2011) The transcription factors Snail and Slug activate the transforming growth factor-beta signaling pathway in breast cancer. PLoS One 6: e26514.
41. Fenouille N, Tichet M, Dufies M, Pottier A, Mogha A, et al. (2012) The epithelial-mesenchymal transition (EMT) regulatory factor SLUG (SNAI2) is a downstream target of SPARC and AKT in promoting melanoma cell invasion. Plos one 7: e40378.

42. Ding X, Park SI, McCauley LK, Wang CY (2013) Signaling between transforming growth factor beta (TGF-beta) and transcription factor SNAI2 represses expression of microRNA miR-203 to promote epithelial-mesenchymal transition and tumor metastasis. J Biol Chem 288: 10241-10253.

43. Sonkoly E, Lovén J, Xu N, Meisgen F, Wei T, et al. (2012) MicroRNA-203 functions as a tumor suppressor in basal cell carcinoma. Oncogenesis 1: e3.

44. Tian L, Li M, Ge J, Guo Y, Sun Y, et al. (2014) MiR-203 is downregulated in laryngeal squamous cell carcinoma and can suppress proliferation and induce apoptosis of tumours. Tumour Biol 35: 5953-5963.

45. Prat J; FIGO Committee on Gynecologic Oncology (2014) Staging classification for cancer of the ovary, fallopian tube, and peritoneum. Int J Gynaecol Obstet 124: $1-5$.

46. Chen Z, Wang Y, Liu W, Zhao G, Lee S, et al. (2014) Doxycycline inducible Krüppel-like factor 4 lentiviral vector mediates mesenchymal to epithelial transition in ovarian cancer cells.PLoS One 9: e105331.

47. Joseph MJ, Dangi-Garimella S, Shields MA, Diamond ME, Sun L (2009) Slug is a downstream mediator of transforming growth factor-beta1-induced matrix metalloproteinase- 9 expression and invasion of oral cancer cells. J Cell Biochem 108: 726-736.

48. Chen D, Zhang Y, Wang J, Chen J, Yang C, et al. (2013) MicroRNA-200c overexpression inhibits tumorigenicity and metastasis of CD117+CD44+ ovarian cancer stem cells by regulating epithelial-mesenchymal transition. $J$ Ovarian Res 6: 50.

49. Guo F, Cogdell D, Hu L, Yang D, Sood AK, et al. (2014) MiR-101 suppresses the epithelial-to-mesenchymal transition by targeting ZEB1 and ZEB2 in ovarian carcinoma. Oncol Rep 31: 2021-2028. 